\title{
Enseñanza-aprendizaje en el taller de diseño
}

\section{Cristina Dreifuss Serrano}

Universidad de Lima, Perú

Recibido: 17 de junio de 2014 / Aprobado: 7 de julio de 2014

Los procesos de transmisión de conocimiento en contextos formales son definidos en términos de enseñanza-aprendizaje, una actividad dual que involucra a los estudiantes y a los docentes, así como al ambiente donde se realiza el proceso. Con este punto de partida, en el artículo se analizarán distintos tipos de enseñanza-aprendizaje dentro del taller de diseño de la carrera de arquitectura. Este es un espacio particular, en el que el estudio de casos es fundamental para el desarrollo de las habilidades del alumno, así como para la transmisión de conocimientos teóricos, entendiendo que el taller de diseño en la carrera de arquitectura es la columna vertebral de la formación del arquitecto en nuestro medio.

taller de diseño, enseñanza, aprendizaje, arquitectura, tipo

\section{The teaching-learning process in the desing workshop}

The process of knowledge transfer in formal contexts are defined in terms of teaching and learning, a dual activity that involves students, teachers, and the environment in which the procedure is performed. With this starting point, different types of teaching and learning into the architectural design workshop courses will be analyzed. This is a particular area in which the case study is critical to the development of students' skills, as well as for the transmission of theoretical knowledge, understanding that the design workshop in a career in architecture is the backbone of architectural education in our country.

workshop design, teaching, learning, architecture, type 
Es claro el estatus del taller de diseño y su importancia dentro de la malla curricular de la Carrera de Arquitectura. En este espacio es donde se generarán las situaciones que busquen enfrentar a los estudiantes con la mayor cantidad de aspectos derivados del ejercicio de la profesión. Goldschmidt sintetiza las funciones del taller de una manera muy escueta, señalando que es el espacio donde se debe forjar la práctica y así asimilar el conocimiento ganando experiencia bajo la guía de un instructor (2003).

Dentro del taller de diseño, el objetivo que se debería perseguir es que el alumno posea las herramientas de diseño (teoría) e intercambio de ideas (crítica), que lo nutrirán de elementos para desarrollar sus propias creaciones.

Lo cierto es que la percepción tanto de los alumnos como de los profesores es, en muchos casos, bastante diferente a las ideas colocadas en blanco y negro por los jefes de taller.

No se puede negar que dentro de los talleres de diseño, la situación aparentemente simple de un grupo de alumnos dirigido por un grupo de arquitectos se ve matizada por una serie de factores.

En primer lugar, el estado del avance académico de los estudiantes al empezar el taller, lo que se traduce en que un alumno de primer ciclo tendrá percepciones y actitudes muy diferentes a las que pueda tener uno de quinto o uno de noveno. Aún dentro de los alumnos de un mismo nivel de base, el grado de madurez y el bagaje de conocimientos y experiencias previas que puedan tener marcará un factor adicional al iniciar el primer ciclo. En un entorno de pluralidad cultural, la procedencia y el nivel socioeconómico de los estudiantes tendrán una repercusión en las diferentes maneras de aproximarse al diseño arquitectónico, las soluciones estéticas que se prefieran, e incluso en la relación que se establezca con los profesores. Finalmente, las particularidades de cada individuo al momento de procesar la información y las experiencias con las que se ve enfrentado plantean retos adicionales a la organización no solo de los talleres sino de la malla curricular en general (Felder \& Silverman, 1988).

Por otro lado, se pueden apreciar diferencias entre los jefes y profesores de taller, debidas a factores tales como la experiencia profesional, el tiempo dedicado a la docencia, su propia formación y las influencias recibidas. 
¿De qué manera estas diferencias se manifiestan en los talleres de diseño? ¿Cuáles son los diferentes enfoques de dichos espacios y cómo estos se articulan entre sí? ¿De qué manera un taller de diseño puede motivar o inhibir las capacidades de estudiantes y docentes?

\section{LA ENSEÑANZA-APRENDIZAJE}

Las diferentes dinámicas que ocurren dentro de un aula se unifican en un solo proceso amplio llamado enseñanza-aprendizaje. De este modo, lo que en algún momento se pensó que eran variables independientes hoy corresponden a un binomio indisoluble en el que si bien se pueden reconocer dos caras, es la unión de estas la que produce los cambios dentro del aula. El concepto de enseñanza-aprendizaje implica, además, que el conocimiento no solo es transmitido de manera unidireccional y vertical, sino que este se genera con la interacción de alumnos y profesores, y que, en un entorno ideal, unos aprenden de los otros de modo bilateral.

\section{MANERAS DE APRE(HE)NDER}

El primer factor que se debe considerar dentro de un aula es la motivación, la cual constituye al mismo tiempo uno de los factores en los que menos injerencia tiene un docente. Evidentemente, existen técnicas, habilidades didácticas y personales del docente, temas y enfoques que pueden aumentar la motivación de los alumnos ${ }^{1}$, pero debemos contar además con la posibilidad de que esta no se dé. Uno de los errores es pensar que el estudiante viene con ganas de aprender. Esta sería la situación ideal, pero en la actualidad, con el crecimiento del sector construcción, las presiones familiares, los nuevos grupos económicos, entre otras razones, es claro que el estudio de una carrera universitaria no es solo un asunto vocacional, sino que se plantea como un signo de estatus.

1 "Poco se ha escrito sobre cómo el cuerpo docente puede mejorar esta interacción [entre estudiantes y profesores] o cómo pueden mejorar la calidad de su enseñanza dentro del taller de diseño" (Ochsner, 2000, p. 194). 
Dejando esta situación particular de lado, la manera como los contenidos son presentados será gravitante para fomentar la motivación en los alumnos y, por lo tanto, para el desarrollo exitoso del taller. La dificultad radica en que no todos aprendemos del mismo modo.

Felder y Silverman (1988) reconocen cinco clasificaciones posibles de los tipos de aprendizaje. Estas clasificaciones se presentan como parejas de opuestos. En la mayoría de personas, un lado de esta dupla predominará más que el otro, lo que lleva a anticipar la mejor manera como las personas asimilarán la información $\mathrm{dada}^{2}$ :

- Aprendizaje sensitivo e intuitivo. En 1921, Carl Jung publica Psychological types (Tipos psicológicos), libro en el que se presentan dos maneras de percibir el mundo. El aprendizaje sensitivo tiene que ver con la observación y la adquisición de información a través de los sentidos. El aprendizaje intuitivo se refiere a la percepción indirecta mediante la especulación, la imaginación o las corazonadas. El primer tipo busca la experiencia directa, el trabajo manual, la experimentación y el estudio de casos, mientras que el segundo prefiere una asimilación inicial de la teoría, desde donde se podrán inferir las particularidades de situaciones específicas.

Todos los individuos usan ambas formas para percibir su entorno, pero en la mayoría de individuos se da con mayor frecuencia uno de los tipos de aprendizaje que el otro.

- Aprendizaje visual, auditivo y kinestésico.Estas tres categorías,o modalidades, se refieren a cómo las personas asimilan mejor la información: de manera visual (diagramas, imágenes, símbolos), auditiva (sonidos, música, palabras) y kinestésica (a través de movimiento, tacto, gusto, olfato). Esta última modalidad tiene que ver no solo con la percepción de la información, sino también con la manera como esta se procesa. No toda la información es adquirida del mismo modo, y a esto se suma la situación adicional de la edad de los alumnos. En muchos casos, las poblaciones jóvenes con métodos de

2 Para mayor información sobre los tipos de aprendizaje (Learning styles) se puede consultar la página central del proyecto, dirigido por la North Carolina State University, en el siguiente enlace: http://www4.ncsu.edu/unity/lockers/users/f/felder/public/lLSpage.html 
estudio aún no consolidados todavía no han descubierto cuál es su manera de aprendizaje más efectiva, y en el transcurso de los primeros años de universidad es posible que se invierta un tiempo considerable en descubrirlo.

- Aprendizaje inductivo y deductivo. Es un tipo de aprendizaje en función a la relación del todo y sus partes. Quienes aprenden de modo inductivo, razonan partiendo de los detalles y son capaces de concluir en las generalidades, es decir, de establecer reglas o constantes frente a un grupo de fenómenos dados. Los individuos con una mayor disposición al aprendizaje deductivo, por el contrario, van a inferir las consecuencias en base a conocer los principios fundamentales de las cosas.

- Aprendizaje activo y reflexivo. La interiorización de la información recibida puede ocurrir de dos formas: a través de la experimentación activa o de la observación reflexiva. En el primer caso, se trata de poner en práctica los conceptos dados en confrontación con situaciones reales o terceras personas, ya sea en una experimentación directa o a través de debates, discusiones, etcétera. El segundo caso se refiere a una postura de interiorización de manera introspectiva, que puede pasar por ensayos, creaciones individuales o simple reflexión personal.

- Aprendizaje secuencial y global. Por lo general, al enseñar se suele presentar los contenidos de manera secuencial, es decir, siguiendo una progresión lógica de eventos según un hilo conductor. Los estudiantes que aprenden de manera global presentan ciertas dificultades para captar los conceptos en medio de esta secuencia, pero pueden captar una serie de ideas al mismo tiempo, al entender de qué manera estas se interrelacionan o forman parte de un todo. Dentro de la enseñanza de la arquitectura en particular se debate sobre las ventajas de un aprendizaje progresivo, que trate los temas uno a la vez, empezando por los más simples hasta llegar a los más complejos. Por otro lado, hay quienes abogan por una presentación total de la profesión que sirva como base a la cual referirse durante el aprendizaje parcial de los aspectos puntuales de la profesión ${ }^{3}$.

3 "I think that beginning students should be exposed to the broadest possible view of architecture to provide a frame of reference for subsequent specific learning [...]. Since beginning students don't possess the knowledge and experience to handle complete architectural problems, a 
Los estudiantes de arquitectura, por sus propias características cognitivas y afectivas, son identificados más con algunos tipos de aprendizaje que con otros. Es probable que, en general, muchos de ellos aprendan mejor de una manera visual que auditiva o verbal, o siguiendo un proceso más activo que reflexivo.

Sin embargo, estadísticamente, dentro del taller esta diversidad de aprendizajes nos colocará frente a más de un tipo de aprendizaje. Esto plantea una situación en la que un mismo método de estudio, un currículo fijo, un plan institucional, no serán suficientes para satisfacer las necesidades de la población total en un salón de clases.

A cada uno de estos tipos de aprendizaje corresponde una serie de recursos didácticos que optimizarán la enseñanza. Por otro lado, no podemos pretender que en un salón de clases universitario se pongan en práctica todos los recursos existentes con el fin de llegar a todos los estudiantes por igual. Esta dificultad exige de los profesores respuestas flexibles y, en algunos casos, incluso individualizadas. Afortunadamente, la organización interna de los talleres de diseño se plantea como el terreno ideal para este tipo de respuestas.

La cantidad de alumnos por profesor no es elevada y la frecuencia de las sesiones, así como su duración, es alta. De este modo, la relación entre alumnos y docentes puede llegar a ser cercana, lo que permite a estos últimos adecuar sus planes de estudio a los retos que cada grupo e, idealmente, cada alumno plantea dentro del taller. Sin embargo, adicionalmente existe otra dificultad dentro del aprendizaje.

La aprehensión de información es una transacción entre lo que está "ahí” físicamente, en el mundo "real", y lo que queremos ver partiendo de los esquemas que nos hemos fabricado con lo que estamos acostumbrados a ver. Por muy "racionales" que intentemos ser, por ejemplo al observar las "tendencias" de las personas, siempre obligaremos a los hechos a encajar en nuestras preferencias personales (Broadbent, 1973, pp. 31-32).

No estamos tratando solo con diversos tipos de aprendizaje, sino con individuos cuyo modo de ver el mundo - ideas preconcebidas, experiencias,

design exercise should emphasize a particular, clearly defined aspect within a larger context" (Simmons, 1978, p. 18). 
referencias, conocimientos previos — varía ampliamente. Esta situación, que puede entenderse como una dificultad adicional al momento de organizar un taller de diseño, es además una fortaleza. La diversidad dentro de un taller nos enfrenta con soluciones plurales, que no solo ofrecen distintas respuestas a un mismo encargo, sino que parten de puntos disímiles entre sí y de supuestos que pueden llevar a experiencias enriquecedoras para el taller.

\section{MANERAS DE TRANSMITIR}

La transmisión de información se articula en torno a dos conceptos: pedagogía y didáctica. El primero se refiere al conjunto de ideas, teorías o conocimientos que se desea transmitir. Parte de la visión del profesor o del conjunto de profesores sobre qué es la arquitectura (el tema por transmitir), y de una toma de partido con respecto a qué particularidades de este universo amplio van a ser enseñadas (los subtemas, enfoques o especialidades).

La primera tarea del docente consiste en determinar cuáles son estas particularidades, cómo deben articularse entre sí y de qué modo esta articulación se da, desde un punto de vista gnoseológico, dentro de la disciplina y, desde un punto de vista administrativo, dentro del currículo de la escuela.

El término "didáctica" define a los métodos empleados por la cátedra para la transmisión de las ideas elegidas. Tiene que ver con los recursos que se van a utilizar — materiales, lecturas, ejercicios, tiempos, criterios de calificacióny con la manera cómo dichas ideas son transmitidas: cómo se comunica el profesor, qué relación establece con los alumnos, etcétera. De la combinación de ambas, pedagogía y didáctica, surge la "propuesta pedagógica". En cuanto a los profesores del taller de diseño y la manera cómo estos enfocan la arquitectura y su enseñanza, Alfano (2000) reconoce cuatro tipos básicos:

- El profesionalista, que parte de concebir al arquitecto dentro de la sociedad productiva, y por lo tanto, enseña en función al rol que debe cumplir (aptitud técnica) ${ }^{4}$.

4 "The educational benefits to the student is a greater awareness and understanding of the importance of the client in the design process. It illustrates the difficulties inherent in communicating effectively with 
- El que vincula su taller con otros "entornos culturales", artísticos o no, como las ciencias sociales, la tecnología, la escultura, el cine, etcétera.

- El que se centra en la creatividad personal y busca desarrollarla.

- El que espera una respuesta "procedente del trabajo teórico y analítico de la arquitectura".

A estos enfoques corresponden metodologías de enseñanza. Nuevamente se presentan grupos de opuestos, pero esta vez se trata de propuestas didácticas sobre cómo transmitir la información deseada. Dichas parejas definen posturas extremas, entre las que cada método se coloca en mayor proximidad a una postura con respecto a la otra.

- Aplicación de una "teoría" predefinida / Definición de un conjunto de principios en cada proyecto de manera específica. En el primer caso se trata de la utilización de una teoría del diseño arquitectónico, que, generalmente, corresponde también a una teoría de la arquitectura. Esta teoría es superpuesta a cada uno de los encargos del taller y se plantea como una suerte de método de diseño universal. Una manera opuesta de dirigir el taller emplea principios de trabajo para cada proyecto de manera particular. El docente, en este caso, se asegura de que el alumno cumpla un recorrido autónomo y con conciencia de sí mismo por medio de la experiencia directa y adquiera, de esta forma, un método de trabajo.

- Aproximación analítica-deductiva / Aproximación ideativa-inductiva. La primera aproximación comprende deducir procedimientos de trabajo específicos a partir de principios generales y teorías, con el fin de obtener modos de intervención a partir de normas generales o generalizables.

La inducción parte de hechos particulares de los cuales se obtienen formulaciones generales. Esta modalidad de trabajo utiliza procesos de composición que no han sido vistos a priori, sino que son "inventados" para cada caso específico.

the client, untrained in architectural methodology or language, and translating that client input into design decisions. The primary objective of using the live client as an educational tool is for broadening the student's realistic understanding of the profession of architecture" (Clay, 1974, p. 24). 
- Docente "mayeuta" / Docente "maestro del oficio". En el primer caso, el docente evita imponer su propia personalidad al estudiante y asume el papel de guía, de manera que este último asuma una capacidad interpretativa y expresiva propia. Se vale de un recurso didáctico que se remonta a la mayéutica socrática, que, independientemente del ámbito cognoscitivo, promueve el crecimiento a través del conocimiento y las facultades individuales para el aprendizaje.

El docente que funge de maestro de oficio, por su parte, afronta los problemas de diseño a través de sus propios conocimientos y experiencia, de su propia manera de hacer el proyecto. En muchos casos, más que una exploración buscará la implementación de los recursos que a él le han funcionado dentro de la práctica, en un aprendizaje que se basa fundamentalmente en su método personal.

La adopción de un determinado enfoque de lo que se va a enseñar (pedagogía) y del tipo de enseñanza que se dará en el taller (didáctica) se plantea como una propuesta que puede satisfacer o no las necesidades grupales y personales de los alumnos.

En muchos casos, la adopción de una propuesta pedagógica es un ejercicio empírico.Los profesores plantean sus talleres en base a sus propias experiencias, tanto en la escuela como en la profesión, y son pocos los casos en los que no solo se da un debate constante entre pares, sino también una capacitación teórica que lo acompañe $e^{5}$.

Existe, además, un componente que, en muchos casos, es determinante. Este tiene que ver con las habilidades personales del profesor y con su capacidad de

5 "En efecto, el argumentar que porque los profesores alguna vez fueron alumnos de un taller de diseño, ellos tienen los conocimientos previos para convertirse en instructores de taller, plantea muchas preguntas; por ejemplo: 1. ¿Son los modelos pedagógicos experimentados cuando fueron estudiantes, los únicos que los docentes tienen para sus propios talleres de diseño? 2. ¿Son estos modelos adecuados para poner al día a los docentes en lo que podrían ser treinta 0 incluso cuarenta años de enseñanza de la arquitectura? 3. ¿Es que realmente no hay alguna otra fuente de información que pueda ayudar a considerar lo que sucede en la interacción entre profesores y alumnos dentro del taller de diseño? La suposición parece ser que cualquiera que ha pasado por un taller de diseño sabrá qué decir y cómo proceder" (Ochsner, 2000, p. 194). 
transmitir información, con el fin de fomentar la motivación en los alumnos, hacer los temas accesibles y, en general, generar una atmósfera dentro del taller que promueva el desarrollo de sus objetivos.

\section{EL TALLER DE DISEÑO}

Definimos como "Taller de Diseño" a cada uno de los cursos en los que el alumno de arquitectura, a lo largo de la carrera, aprende sobre las tareas proyectuales del arquitecto desde un enfoque práctico. Este taller es, en la mayoría de casos, planteado como un "ensayo de vida profesional", y su desarrollo propone tareas en los que se busca que el futuro arquitecto ponga en práctica de modo paulatino las habilidades que requiere el ejercicio de la profesión.

Los talleres constituyen la espina dorsal de la carrera de arquitectura. A cada ciclo académico corresponde un taller de diseño y estos se suceden de manera lineal. Del mismo modo, el nivel de complejidad y dificultad del trabajo realizado en ellos es progresivamente ascendente.

Se trata de un modelo pedagógico globalmente utilizado, que recibe críticas constantes en las que se señala su arbitrariedad, falta de rigor teórico y alto predominio de lo subjetivo y personal como sus principales carencias. Sin embargo, es al mismo tiempo un espacio para la experimentación, el fomento de la creatividad y la enseñanza en base a la resolución de problemas complejos (Ledewitz, 1985).

Ciertamente, comparado con escenarios de clase típicos, los talleres de diseño son lugares activos donde los estudiantes participan intelectual y socialmente, en un constante cambio entre posturas analíticas, sintéticas y evaluativas, a través de conjuntos diferentes de actividades (dibujo, discusión, elaboración de maquetas). El hecho que estos atributos sean característicos de muchos talleres de diseño es claro, y testifica la unicidad del taller como vehículo para la educación del estudiante.

La intensidad y el compromiso en cuanto al tiempo que el taller requiere es el resultado de la naturaleza de lo enseñado. El diseño de un edificio es una suerte de problema de múltiples variables, que no puede ser resuelto con un proceso de deducción lógica, o con la aplicación de una serie de fórmulas aprendidas. En la mayoría de los otros cursos, cuando se plantean problemas, los estudiantes 
saben que una solución o un tipo de soluciones ya han sido determinados. Cuando se presentan los problemas, estos son usualmente resueltos con la aplicación de un cuerpo de conocimiento ya adquirido, según un método aprendido. En contraste, el taller de diseño ofrece problemas sin resultados conocidos. Algunos aspectos técnicos del problema de diseño pueden ser predecibles dentro de ciertos límites (es por eso que tenemos estudios de tipología, composición y similares) pero se espera que la solución precisa sea original (Ochsner, 2000, p. 195).

Dentro de la situación del taller suelen regir dos escalas de valores. Por un lado, el currículo manifiesto, es decir, los contenidos temáticos del taller y el modo como estos son administrados. En algunos casos, su descripción es clara, e incluye los criterios de calificación con el fin de que los estudiantes sepan desde el inicio cuales son los objetivos.

Sin embargo, existe en paralelo la situación del currículo oculto, es decir, el conjunto de valores, actitudes y normas no manifiestos dentro del taller, pero que se revelan conforme al avance de este. En algunos casos, los mismos profesores no son conscientes de esto; en otros, se considera que el hecho de que el alumno los vaya descubriendo de a pocos es una habilidad que se debe desarrollar. "En comparación al currículo formal que enfatiza el conocimiento [...] el currículo oculto se focaliza en cuestiones que conciernen a la ideología de dicho conocimiento, y las prácticas sociales que estructuran las experiencias de estudiantes y profesores" (Dutton, 1987, p.17). Dentro de los contenidos manifiestos, podemos reconocer que la forma como se dicta un taller de diseño y sus contenidos están determinados por la combinación de tres factores:

- Las directivas de la Facultad de Arquitectura, a través del decanato o la dirección de carrera. En algunos casos, estas directivas son discutidas a través de consejos de facultad periódicos, o reuniones entre los profesores y las autoridades. En otros casos, se adscriben a un corpus teórico previamente definido que puede apoyarse en ideas tomadas de otras escuelas.

- La posición o nivel que ocupa el taller de diseño en relación con toda la carrera, es decir, los talleres iniciales en los primeros ciclos, los intermedios y los talleres avanzados hacia el final de la carrera. La mayoría de escuelas asigna para cada nivel un conjunto de objetivos y tareas específicas, los que 
serán completados por los estudiantes antes de ser considerados aptos para pasar al siguiente nivel.

- El enfoque particular del profesor o de los profesores a cargo del taller. Dependiendo de la flexibilidad de la estructura en la escuela, y de la cantidad de profesores del taller, este enfoque puede ser el fruto de la discusión de grupos pequeños de docentes, o el producto de discusiones en los que uno, el jefe de taller, marca la pauta que se debe seguir.

Estos tres aspectos pocas veces se encuentran equilibrados. De acuerdo con las características propias de cada facultad, uno tomará una mayor preponderancia frente a los demás. En las escuelas antiguas, por ejemplo, el peso de las directrices de las facultades suele ser mayor; por otro lado, en las escuelas con esquema de talleres horizontales puede darse que el tercer punto cobre una mayor importancia.

\section{enseñanZa-Aprendizaje en el taller de diseño, una tipología}

Los talleres de diseño pueden agruparse en enfoques, es decir, en aquel o aquellos aspectos de la arquitectura a los que se dan mayor importancia durante el desarrollo del taller. Es posible que un taller tenga más de un enfoque, sobre todo en los talleres altos, donde se busca que los estudiantes integren los distintos aspectos del hecho arquitectónico. En opinión de Segret, "la pedagogía en arquitectura es más un complejo, pero controlado, conjunto de relaciones que constituyen geografias sobre las superficies de paisajes culturales, que una superposición de funciones en un lugar"'(Segrest, 1991, p. 11, traducción mía). Frente a esta realidad integradora, ¿por qué es necesario proponer una clasificación? En primer lugar porque la comprensión de las partes y sus posibles interrelaciones nos permitirían una percepción más clara del todo. Por otro lado, dado el carácter pedagógico de los talleres de diseño, estos deben apuntar a una forzada simplificación que lleva a la profundización de ciertos aspectos de la arquitectura. De este modo, los alumnos podrán enfatizar en un número limitado de tareas a la vez, lo que permitirá consolidar paulatinamente el aprendizaje e ir integrando poco a poco el resto de variables. 
Los enfoques aquí expuestos se refieren, en términos generales, a aproximaciones a una definición de arquitectura ampliamente aceptadas y difundidas (Quaroni, 1987; Muñoz,2004;Solá-Morales,Llorente,Montaner,Ramón, \& Oliveras, 2006). Se busca sistematizar, en un número limitado de "conceptos fundamentales", los diversos aspectos del diseño arquitectónico para trasladarlos luego a su enseñanza. Después de un proceso de observación que abarcó talleres de diseño en distintas universidades ${ }^{6}$, se definen seis enfoques predominantes:

\section{LA MATERIALIDAD: EL ENFOQUE TÉCNICO-TECTÓNICO}

La dimensión técnica suele considerarse de dos maneras diferentes. Pueden tomarse los materiales como punto de partida, hablando de «construcciones de madera» y "construcciones de hormigón armado», clasificación que se emplea en la práctica arquitectónica y aparece en muchos manuales. Pero también pueden tomarse como punto de partida los elementos de la construcción. La construcción nos proporciona cimientos, paredes, suelos y techos, escaleras, puertas y ventanas. Todas estas partes pueden ejecutarse en diversos materiales.

(Norberg-Schulz, 2001, p. 104).

Tectónica, de origen griego (la palabra tekton designa al constructor), es el conjunto de aspectos de cualquier obra arquitectónica referidos al sistema constructivo, y por ende a los materiales y su lenguaje. La técnica aplicada a este enfoque sería la manera como este sistema constructivo se pone en práctica y funciona, cuáles son las lógicas de los materiales y sus posibilidades inherentes.

Un taller con este enfoque parte de la arquitectura como construcción. Es un objeto real, sometido a las leyes de la física y, por lo tanto, debe ser realista en cuanto a la manera de construirse, el sistema utilizado, la transmisión de

6 El estudio de casos se inició en el marco de la tesis de la autora "La enseñanza de Arquitectura en el primer Taller de Diseño. Análisis crítico y propuestas" (Lima: Universidad Nacional de Ingeniería, Facultad de Arquitectura, Urbanismo y Artes; Sección de Postgrado y Segunda Especialización, 2008). Esta etapa inicial consideró los talleres de diseño de las escuelas de arquitectura en la Universidad Nacional de Ingeniería, la Universidad Ricardo Palma, la Universidad Peruana de Ciencias Aplicadas y la Pontificia Universidad Católica del Perú. Posteriormente se incluyó la producción de los talleres de la Carrera de Arquitectura de la Universidad de Lima. 
cargas, etcétera. La producción dentro del taller puede, además, incorporar las habilidades manuales y la destreza de los alumnos como constructores.

Otra opción es el estudio más bien teórico de las capacidades de los diferentes materiales. De esta manera se introduce al alumno en los conceptos estructurales de tracción, compresión, transmisión de cargas, entre otros.

En este enfoque, la creatividad es canalizada por las limitaciones de cada material; se busca que el alumno pueda responder, con una lógica constructiva determinada, a las diferentes situaciones compositivas que se plantean.

Ejemplos de estos enfoques son los talleres en los que se trabaja a escala 1:1 (en general, en los niveles iniciales), los que exploran con el uso de un material determinado en los trabajos del taller, los que proponen la premisa de diseñar con un sistema constructivo determinado o, simplemente, los que exigen de sus alumnos no solo una respuesta formal, sino también un correlato estructural, en un mayor o menor grado de desarrollo (niveles avanzados).

El enfoque técnico-tectónico va a forzar al alumno a circunscribir sus soluciones formales en el marco de "lo que puedo construir". Las formas completamente libres, que carecen de lógica estructural o que no pueden construirse con el material o el sistema propuesto están, por lo tanto, fuera de su alcance.

Esta realidad es vista por algunos como un aspecto positivo, pues fuerza al alumno, desde el inicio, a entender que la arquitectura tiene como objetivo final su realización y que, por ende, el diseño debe estar subordinado a criterios de realidad. Otros consideran, en cambio, que, en especial en los niveles iniciales, no se debería constreñir la creatividad del alumno con aspectos constructivos que van a limitar su potencial creativo.

La oposición a esta postura tendrá como resultado, en el mejor de los casos, formas fantásticas, irreales y que, probablemente, difieran en gran medida de las imágenes con las que el estudiante está familiarizado. Esta libertad formal en la creación será producto de una serie de ejercicios cuyo objetivo es, en muchos casos, opuesto al enfoque técnico-tectónico: explorar con las formas más imaginativas posibles. Se supone, entonces, que el estudiante adquirirá los criterios estructurales y de comportamiento de los materiales en etapas posteriores de la carrera. 


\section{EL ESPACIO ARQUITECTÓNICO}

De forma constante, nuestro ser queda encuadrado en el espacio. A través del volumen espacial nos movemos, vemos las formas y los objetos, oímos los sonidos, sentimos el viento, olemos la fragancia de un jardín en flor.En sí mismo carece de forma. Su forma visual, su cualidad luminosa, sus dimensiones y su escala derivan por completo de sus límites, en cuanto están definidos por elementos formales. Cuando un espacio comienza a ser aprehendido, encerrado, conformado y estructurado por los elementos de la forma, la arquitectura empieza a existir.

(Ching, 1995, p. 108).

La locución "espacio arquitectónico" ha sido definida de diversas maneras; dentro de la enseñanza de la arquitectura es difícil de entender en un primer momento, y más aún, dificil de explicar. Los estudiantes, dentro de la realidad sobremediatizada, conocen sobre arquitectura a partir de imágenes de distintas procedencias. "En la época de la reproducción mecánica y la manipulación electrónica de imágenes, la experiencia de la realidad física de la arquitectura viene precedida, sustituida o inmersa en una avalancha de imágenes brillantes" (Piotrowski, 1996, p. 104).

La enseñanza de la arquitectura que parte del espacio arquitectónico busca, entonces, contrarrestar esta tendencia y mostrarla al estudiante a través de la experiencia espacial.

Este enfoque tiene dos aproximaciones. Por un lado la intención de descubrir, de poner en contacto al estudiante con las diferentes percepciones que recibimos en espacios con diferentes configuraciones, y en las sensaciones que producen dichos lugares. Por otro lado, el crearlo; el poder plasmar en un objeto arquitectónico una determinada sensación, a través de la concepción de espacios ad hoc.

La dificultad reside en que los alumnos no saben entender el espacio y, por lo tanto, no logran "crearlo". Las características del término, fundamentalmente abstracto, la diferenciación entre sus significados coloquiales y lo que este significa en la arquitectura, la dificultad de describir con palabras la sensación que produce el estar en un determinado lugar son solo algunas de las situaciones a las que nos enfrentamos al hablar del "espacio arquitectónico". 
Una aproximación al tema, eminentemente teórica, es la de "clasificar" el espacio según sus características. Surgen entonces los sistemas compositivos, tales como "espacio lineal", "diagonal", "espacio nucleado". Otro enfoque consiste en realizar construcciones en escala 1:1. De esta manera se establece un diálogo con el alumno sobre un lugar común, que tanto este como el profesor pueden percibir casi de la misma manera.

La dificultad de la primera propuesta radica en que se trabaja con objetos a escala. El alumno no trabaja con un "espacio lineal", sino con una representación de este. En este contexto, cabe la posibilidad de que no exista un entendimiento real entre el profesor y el alumno.

El primero no tiene un real control de lo que el alumno está percibiendo, y las representaciones en maqueta de este pueden limitarse a composiciones que "siguen la forma", mas no la lógica de la composición espacial. La habilidad de representar un "espacio lineal" en escala 1:50 puede ser fruto de un adiestramiento más que de una comprensión cabal de lo que se busca representar.

En la segunda propuesta, la de construcciones en escala 1:1, surgen limitaciones impuestas por las habilidades constructivas de los propios estudiantes y por las dimensiones, en ocasiones reducidas, de los espacios que se están construyendo.

Puede darse que, debido a las características de los materiales, la calidad espacial de muchos de los objetos sea similar, y los conceptos por aprender sean, por lo tanto, limitados.

En general, trabajar en base al concepto de espacio arquitectónico exige una serie de referentes reales, que pueden ser construcciones existentes, conocidas por profesores y alumnos, a las cuales unos y otros puedan referirse, o el método aún más directo de las visitas de campo.

Es un entrenamiento que se enfoca en la percepción del futuro arquitecto, y en la comprensión de las capacidades de la arquitectura para generar sensaciones a través de las diferentes posibilidades en el interior de los edificios. 


\section{LA COMPOSICIÓN FORMAL}

El análisis formal consiste en indicar elementos y relaciones; esto significa, en primer lugar, que debemos emplear como dimensiones de comparación (elementos) objetos definidos y, en segundo lugar, que habría que hacer un balance de las interrelaciones entre estas dimensiones.

(Norberg-Schulz, 2001, p. 90).

Esta propuesta ofrece una amplia variedad de soluciones e implementaciones metodológicas y es, posiblemente, el enfoque que siempre estará presente. La mayoría de talleres, al mencionar sus preocupaciones centrales, se refieren tarde o temprano a la forma o el aspecto externo de la producción de los alumnos.

La diversidad de soluciones tiene que ver con los criterios dados por el taller para la valoración formal o estética de un determinado objeto. La principal dificultad de esta aproximación está en definir las razones cualitativas que determinan que un objeto "se vea bien" o no. Actualmente, los criterios estéticos y los juicios de valor a partir de la forma se alejan cada día más de los cánones preestablecidos, y el culto a la individualidad y a la originalidad dificulta en gran manera un diálogo con respecto a la calidad formal de una creación individual.

Una de las propuestas está ligada a la antropometría desde el punto de vista de la proporción. En esta se parte de las dimensiones del ser humano como ideal de proporción y, sobre todo, de las relaciones entre las partes y el todo. Este estudio permite que el alumno comprenda, en base a un referente tangible, cuándo un objeto puede percibirse como desproporcionado o "desbalanceado".

Otra propuesta tiene que ver con las posibilidades de deformación de la masa: adición, sustracción, rotación, etcétera (Eisenman, 2011; Hejduk y Henderson, 1988). De esta manera, la creación formal deja de ser un acto intuitivo y empírico, y se traslada a un plano racional en el que el alumno debe ser consciente de todo el proceso teórico que lleva a una determinada forma. Estas categorías, además, permiten establecer relaciones a priori de "qué cosa queda bien con qué otra" y potencia que, por medio de una serie de pequeños ejercicios, el alumno tenga contacto con la mayor cantidad posible de variaciones de un cubo, que luego podrán ser trasladadas a otro elemento. 
Una variante de este sistema tiene que ver con los principios ordenadores, "artificios visuales que permiten la coexistencia perceptiva y conceptual de varias formas y espacios de un edificio dentro de un todo ordenado y unificado" (Ching, 1995, p. 332). La creación dentro del taller va a enfocarse en estas relaciones entre las diferentes partes y el todo. Dichas relaciones pueden ser sistematizadas en conceptos abstractos — eje, simetría, ritmo, etcétera- que buscan que el estudiante entienda que la composición volumétrica y formal obedece a órdenes racionales. Finalmente, se debe considerar las propuestas más volátiles, en las que la apariencia externa del objeto tiene un valor, pero que, en el transcurso del taller, no se hacen explícitos los criterios que definen que determinada solución "es mejor" que otra.

El peligro de esta actitud, en la que el currículo oculto cobra relevancia, es el de caer en preferencias formales que no podrán ser explicadas luego a los alumnos, y por lo tanto, en que las calificaciones puedan interpretarse como fruto del capricho.

Ocurre entonces que al calificar la producción del taller inicial desde una aproximación formal, se puede caer en soluciones subjetivas que dependan del gusto personal de profesores y alumnos. La crítica como recurso didáctico deberá, en la medida de lo posible, apartarse de criterios volátiles e intentar que el alumno sintonice con criterios compositivos racionales, que puedan ser discutidos y probados de manera racional.

\section{EL USUARIO: COMPORTAMIENTO Y FUNCIÓN}

Todo lo que haga el arquitecto debe ser, sobre todo, la expresión de una institución del hombre, antes de que se convierta en edificio. Uno no sabe lo que el edificio es en realidad a menos que se mantenga una creencia tras el edificio, una creencia en su identidad y en el modo de vida de los hombres.

(Kahn, 2004, p. 96).

Se trata de un enfoque que parte de entender la arquitectura como albergue o escenario de las actividades humanas. Por lo tanto, sus características formales y espaciales dependerán directamente del uso que se dé al espacio en cuestión, es decir, del aspecto funcional. 
Lo funcional no debe entenderse como un conjunto de habitaciones, un organigrama o un flujograma, sino, en un sentido más amplio, como el conjunto de movimientos, actitudes y actividades del ser humano a realizarse en el espacio, y a ser albergados por la forma. La arquitectura, señala Dewey, "expresa además los valores perennes de la vida humana colectiva. 'Representa' las memorias, esperanzas, miedos, propósitos y valores sagrados de aquellos que construyen con el fin de albergar" (2005, p. 230).

En cierta medida, este enfoque está presente en casi todos los talleres donde, en algún momento del semestre académico, se plantea ejercicios en los que se debe trabajar con los alumnos temas de circulaciones, escaleras y relaciones funcionales.

Una manera de enfocar el tema es a partir del movimiento. Aunque este no corresponda a una actividad racional o convencional del individuo, se entiende que el diseño debe albergar el recorrido realizado por él, y el objeto arquitectónico será el producto de la vectorización (abstracción geométrica) de diversos cambios de posición. Esta postura tiene una relación directa con temas de antropometría, pero incorpora la variable adicional del movimiento. Otro enfoque, más cercano a la idea de función, parte de ejercicios realizados en torno al hábitat y busca que los estudiantes descubran las dimensiones de las cosas en base a las funciones básicas del cuerpo humano, y mediante la reinterpretación de los espacios propios de una vivienda: "espacio de comer", "espacio social". Un primer peligro de esta aproximación es llevar a los estudiantes a la impresión errada de que el hacer arquitectura tiene que ver con el uso adecuado de manuales y dimensiones, y se deja de lado aspectos compositivos, formales y espaciales.

Por otro lado, el empleo de estandarizaciones, si no va acompañado de una reflexión crítica, conlleva a designar al ser humano como un arquetipo, sin tener en cuenta particularidades como las diferencias de tamaño de ambientes y objetos de acuerdo a la raza, las distintas interpretaciones de la vivienda contemporánea que dependen de las nuevas actividades del ser humano o de su comportamiento social.En suma, se puede caer en formulismos que, mal entendidos por los alumnos, llevan a creer que con solo la aplicación de algunos preceptos preestablecidos se pueden resolver los diferentes requerimientos arquitectónicos. 


\section{EL SER HUMANO: ANTROPOMETRÍA}

Los sistemas antropomórficos de proporcionalidad se basan en las dimensiones y proporciones del cuerpo humano. [...] No persiguen unas razones abstractas o simbólicas, sino unas razones funcionales. Se proclama, en teoría, que las formas y los espacios arquitectónicos son contenedores o prolongaciones del cuerpo humano y que, por lo tanto, deben venir determinados por sus dimensiones.

(Ching 1995, p. 324).

El sistema Modulor, ideado por Le Corbusier en las décadas de 1930 y 1940, es solo uno de los muchos ejemplos que buscan sistematizar los tamaños de las cosas con el fin de lograr estandarizaciones que redunden en una reducción de costos y una mayor eficacia en los modelos de producción.

Los sistemas de medidas estandarizadas, que se centran en el ser humano y sus proporciones, son producto de una preocupación por humanizar esta industrialización y buscan acercar al hombre a los objetos que lo rodean. En esta perspectiva, la arquitectura, como refugio del hombre, debe responder a sus necesidades no solo de uso sino también de dimensión.

La toma de las medidas del cuerpo humano como punto de partida permite que el alumno sea consciente de dos aspectos: el tamaño de las cosas en relación con su propio cuerpo (la altura de una silla, el alto de una puerta, el ancho de un pasillo) y la idea de proporción como relación de las partes con el todo (la relación entre el largo de una pierna y el de todo el cuerpo, por ejemplo).

El primer aspecto genera un nexo entre el alumno y el espacio que lo rodea, en la medida en que este identifique las relaciones dimensionales con respecto a sí mismo. Posteriormente, las medidas de las cosas se incorporarán al diseño, lo que redundará en creaciones cómodas (lógicas) a la medida del ser humano, como por ejemplo el diseño de escaleras con una adecuada altura de los contrapasos.

La exploración antropométrica lleva al alumno al descubrimiento de una suerte de "reglamento de edificaciones", similar al elaborado por Neufert, en el que el cuerpo humano es el protagonista de las medidas del espacio arquitectónico y sus elementos. Al partir de esta premisa, los diseños resultantes contemplarán, de manera empírica, las proporciones necesarias para la realización de determinada actividad. 
El segundo aspecto de esta exploración mediante la antropometría redunda en temas formales. Frente a la dificultad de evaluar la estética de un objeto con criterios objetivos, tendemos a recurrir a las proporciones. Así, hablamos de objetos esbeltos, extendidos, simétricos. Estas categorías son la síntesis automática de observar las relaciones de las diferentes partes del objeto. Sin necesidad de emular las proporciones de brazos, manos y torsos, el estudio de estas permite una aproximación a las ideas de equilibrio compositivo, y, al mismo tiempo, hace posible la comprensión de criterios estéticos.

El movimiento y su estudio, explicados en la categoría anterior, son también referentes válidos para una aproximación antropométrica al usuario. No se trata solo de las proporciones de un objeto en reposo, sino del espacio que le ocupa al individuo la realización de determinadas acciones.

\section{EL ENTORNO: EL TERRITORIO}

Necesitamos enseñar conocimientos acerca del entorno cotidiano. Cómo éste se estructura, qué podemos aprender de evidencias históricas y contemporáneas, qué diferentes ejemplos se pueden comparar, cómo ésta se comporta a lo largo del tiempo y responde al cambio de uso u otras circunstancias. Enseñar diseño arquitectónico sin enseñar cómo funciona el entorno es como enseñar a estudiantes de medicina el arte de sanar sin decirles cómo funciona el cuerpo humano.

(Habraken, 1962, p. 46).

Las variables compositivas o interpretativas, así como el encargo arquitectónico, se sitúan en un lugar concreto. El trabajo del estudiante, entonces, no se encuentra aislado o presente en un territorio abstracto e irreal, sino que tiene un lugar en un terreno y la relación que establezca con este exterior será fundamental para enriquecer la creación. "Antes de empezar a diseñar, el arquitecto debe reconocer que el 'mundo real' le impondrá ciertas limitaciones. El solar está 'ahí', es un terreno, y sus características pueden deformarse si se convierte en un 'modelo' abstracto cualquiera' (Broadbent, 1973, p. 398). Se puede entender el producto del diseño arquitectónico —el objeto- como un ente en relación simbiótica con su entorno —el contexto. 
Se debe mencionar que al referirnos al entorno o al territorio, son muchas las aproximaciones posibles. Hablamos, entonces, de un entorno urbano inmediato: la calle, el barrio, la plaza, el distrito; un entorno más amplio, un territorio, la ciudad, la región. Por otro lado, podríamos referirnos a la realidad socioeconómica o cultural, como una suerte de "territorio" en el cual insertarnos. Finalmente, el territorio es, también, el paisaje como medio natural.

La postura diametralmente opuesta se da en la metodología de tramas y terrenos abstractos. No solo se trata de un territorio artificial (generalmente cuadrado, subdividido en módulos), sino que incluso los criterios compositivos se enfocan desde el interior, sin considerar lo que pueda ocurrir más allá de los linderos.

En muchos talleres, en especial los más avanzados, los primeros ejercicios toman el entorno como punto de partida. Se busca que los estudiantes analicen las diferentes variables en un entorno dado y en base a estas vean a la arquitectura como un hecho contextual, como una respuesta a las características de un lugar; "la arquitectura se constituye con la ciudad, y con la ciudad se constituyen a la vez las viviendas y los monumentos” (Rossi, 1977, p. 203).

\section{IDEAS FINALES}

La gran mayoría de profesores de arquitectura no poseen formación docente alguna, y su presencia como profesores de taller se debe a una destacada trayectoria profesional o académica (a veces en el campo de la investigación), o incluso, a una suerte de aprendizaje dentro del taller, donde se empieza como asistente y se va ascendiendo puestos hasta lograr ser profesor principal. Por eso los principios pedagógicos, si están presentes, han sido adquiridos de manera empírica, en base a un proceso de ensayo-error y acumulación de experiencias.

En muchos casos, el taller es una suerte de mecanismo vivo, que va cambiando y ajustándose en la medida en que una idea u otra prueben su validez al ser puestas en práctica con los alumnos. Esta actitud es, en principio, positiva, pues obliga a una constante reflexión sobre el camino recorrido y a una sana postura crítica del trabajo en el interior del taller. Sin embargo, esta dinámica de cambios y replanteos puede caer en una suerte de exceso de empirismo, en el que la carencia de una estructura base y de una revisión y análisis de las 
experiencias previas deviene en una reincidencia de ciertos errores o en la pérdida de experiencias e información valiosa.

Los alumnos vienen con un bagaje, con un caudal de conocimientos y experiencias previas que —errados o acertados - afectarán en su desempeño en el aula. Para algunos profesores esta es una circunstancia afortunada, puesto que da a los alumnos, en conjunto, un referente común a partir del cual se puede comenzar a reflexionar sobre la arquitectura. Para otros, en cambio, esta es una circunstancia negativa, puesto que constriñe la capacidad de respuesta de los alumnos; el profesor, entonces, debe propiciar un nuevo inicio, sin este tipo de referentes. Llevado a la exageración, se buscará hacer del alumno una tabula rasa al eliminar todas las ideas previas que puedan haber concebido. Sin embargo, una postura que sobrevalore el bagaje inicial y las soluciones espontáneas de los alumnos, puede ser también nociva al caer en un aprecio excesivo por las respuestas "originales" del estudiante; se cae, entonces, en una falsa realidad en la que todo parece ser maravilloso. El exceso de libertad llevará a una confusión del mismo estudiante frente a la amplitud de posibilidades; este vacío será llenado con el recurso que más se tiene a mano: la imitación. Se incorporarán soluciones previamente vistas del entorno inmediato — casa, barrio, ciudad de procedencia— o de revistas y libros de arquitectura.

Lograr este equilibrio entre las directrices y "pies forzados" dados por la cátedra, y la incorporación de las ideas propias y la creatividad inherente de los estudiantes es una de las principales tareas de la cátedra y los logros obtenidos a este respecto deben ser constantemente debatidos y revisados.

El profesor se encuentra frente a un doble problema profesional. Por un lado, tiene la tarea de transmitir a sus estudiantes una selección adecuada de la cultura de la sociedad de su tiempo; por otro, debe hacerlo respetando la libertad de aprendizaje de sus alumnos:y debe respetarla no tanto por razones morales — que tienen su propio peso, si asumimos que es inmoral constreñir la libertad de la persona humana-, sino por razones prácticas: porque si no se respeta esta libertad —es decir, la espontaneidad de las motivaciones y la capacidad de elegir-, el aprendizaje no tendrá lugar (Laporta, 1993).

Considerando que, sobre todo en los talleres bajos, el contacto de los alumnos con la profesión ha sido prácticamente nulo, los primeros encargos por realizar tienen que ver no solo con el inicio del proceso de creación, 
sino que incorporan la adquisición de un lenguaje arquitectónico, de un método de trabajo dentro y fuera del taller y, finalmente, de una exploración personal sobre las propias ideas y cómo manifestarlas ${ }^{7}$. En un taller en el que este conjunto de "ritos de iniciación" no sea tomado en cuenta, el estudiante, frente a la necesidad de avanzar al ritmo del taller, irá adquiriendo por propia iniciativa los conceptos, las palabras y las herramientas que necesita.

En muchos casos, el alumno, lejos de iniciar un proceso de descubrimiento, se sentirá frustrado y no comprendido por el profesor. Esta situación lleva a que los trabajos elaborados en el interior del taller tengan por objetivo una nota aprobatoria, y no una verdadera búsqueda de conocimiento. Finalmente, algunos alumnos llegarán al punto de abandonar la carrera frente a esta falta de espacios de diálogo con la cátedra. El no establecer un lenguaje común y un conjunto de códigos y criterios manejados tanto por los alumnos como por los profesores dentro del taller, plantea una brecha que deviene en producciones hechas "a gusto del profesor". La falta de criterios de clasificación, determinados por la cátedra y conocidos por los alumnos, genera una situación similar. Ya sea que "la nota" se base en juicios de valor con respecto al producto concluido, participación de los alumnos durante las clases, el avance del trabajo a lo largo del ciclo, categorías estéticas u otros factores, los profesores deben establecer criterios de calificación mensurables y concretos, que eviten posturas en las que el alumno no sepa discernir entre lo "correcto" y lo "equivocado" frente a determinado ejercicio.

Es indispensable señalar que en el interior de los talleres los procesos creativos son tan importantes como la producción en sí. Algunos talleres hacen especial énfasis en que el estudiante adquiera una metodología de diseño en la que este no es producto de la intuición o el gusto personal, sino que responde a una serie de variables, previamente establecidas por el alumno o el contexto. La idea final es que esta metodología sea adoptada por el alumno de modo que, en adelante, pueda enfrentarse a cualquier

7 "El taller es el método primordial para enseñar al menos tres aspectos básicos dentro de la educación en el diseño. Es donde los estudiantes aprenden y practican un conjunto de nuevas destrezas, como visualización y representación, además aprenden un nuevo lenguaje. [...] Palabras como 'forma' y 'escala' tienen significados nuevos y complejos que no son fáciles de internalizar. En tercer lugar, y de un modo muy significativo, el taller es donde los estudiantes aprenden a 'pensar arquitectónicamente'. En arquitectura, como en otros campos, este ' modo de pensar' se refiere a un particular dominio de problemas y soluciones que caracterizan y son fundamentales para el desempeño profesional" (Ledewitz, 1985, p. 2). 
proyecto o ejercicio arquitectónico con una base. Las dificultades surgen cuando no existe una diferenciación clara entre "metodología" y "fórmula". Los estudiantes llegan a la impresión errónea de que la solución de un problema arquitectónico es la aplicación de ciertas reglas invariables; hasta que no se topen con un taller en el que la "fórmula" no sea aplicada, se va a mantener esta visión parcial del cómo se diseña.

\section{REFERENCIAS}

Alfano, F. (2000). Trasmissibilità e insegnamento del progetto di architettura. L'esperienza della Scuola di Palermo. Napoli: Clean Edizioni.

Broadbent, G. (1973 [1971]). Metodología del diseño arquitectónico. En G. Broadbent, Metodología del diseño arquitectónico (21-36). Barcelona: Gustavo Gili.

Broadbent, G. (1973 [1971]). Notas sobre la metodología del diseño. En G. Broadbent, Metodología del diseño arquitectónico (397-414). Barcelona: Gustavo Gili.

Ching, F. (1995). Arquitectura: forma, espacio y orden. Barcelona: Gustavo Gili.

Clay, E. H. (1974). The use of a "client" as a design teaching tool. Journal of Architectural Education, 1-2(28), 23-24.

Corbusier, L. (2008 [1923]). Towards an architecture. Londres: Francis Lincoln Limited.

Dewey, J. (2005 [1934]). Art as esperience. Nueva York: Penguin.

Dutton, T. A. (1987). Design and Studio Pedagogy. Journal of Architectural Education, 1, 16-25.

Eisenman, P. (2011). Diez edificios canónicos: 1950-2000. Barcelona: Gustavo Gili.

Felder, R. M., \& Silverman, L. K. (1988). Learning and teaching styles in engineering education. Engineering Education, 7, 678-681.

Goldschmidt, G. (2003). Expert knowledge or creative spark? Predicaments in design education. Expertise in design. Design thinking research. Symposium 6. Sydney. 
Habraken, N. J. (1962). Soportes: una alternativa al alojamiento de masas. Madrid: Alberto Corazón Editor.

Hejduk, J., \& Henderson, R. (1988). Education of an architect. The Irwin S. Chanin Schooil of Architecture of the Cooper Union. Nueva York: Rizzoli.

Kahn, L. I. (2004 [2000]). Conferencia en el Politécnico de Milán. En A. Muñoz Cosme, Iniciación a la arquitectura. Barcelona: Mairea/Celeste.

Laporta, R. (1993). Natura e finalità dell'educazione. En A. Canevaro, F. Frabboni, E. Frauenfelder, R. Laporta, \& M. Pinto, Fondamenti di pedagogia e di didattica. Roma: Laterza.

Ledewitz, S. (1985). Models of design in studio teaching. Journal of Architectural Education, 2(38), 2-8.

Muñoz Cosme, A. (2004 [2000]). Iniciación a la arquitectura. Barcelona: Mairea/Celeste.

Norberg-Schulz, C. (2001 [1979]). Intenciones en arquitectura.Barcelona: Gustavo Gili.

Ochsner, J. K. (2000). Behind the mask: a psychoanalytic perspective on interaction in the design studio. Journal of Architectural Education, 4(53), 194-206.

Piotrowski, A. (1996). Beginning design and culture. European Association of Architectural Education, 104.

Quaroni, L. (1987 [1977]). Proyectar un edificio. Ocho lecciones de arquitectura. Bilbao: Grafo.

Rossi, A. (1977). Para una arquitectura de tendencia. Escritos 1956-72. Barcelona: Gustavo Gili.

Segrest, R. (1991). Design and the pedagogical text (Excess Bagage). Journal of Architectural Education, 1(45), 11-14.

Simmons, G. B. (1978). Analogy in design: studio teaching models STOR. Journal of Architectural Education, 3(31), 18-20.

Solá-Morales, I., Llorente, M., Montaner, J. M., Ramon, A., \& Oliveras, J. (2006). Introducción a la arquitectura. Conceptos fundamentales. Barcelona: Ediciones UPC. 